\title{
Diverse ligand-binding domain combinations at the distal end of bacterial RTX adhesins are postal codes for biofilm formation.
}

Tyler D. R. Vance, Shayan Assaie-Ardakany, Adam P. Sage, Brigid Conroy, Phil Guo, Corey A. Stevens, Laurie A. Graham and Peter L. Davies

\section{Queen's University}

Many Gram-negative bacteria produce Repeats-In-Toxin adhesion proteins (RTX adhesins) to facilitate microbial adhesion to a variety of biotic and abiotic substrates. These large, multi-domain proteins anchored on the outer membrane share a common architecture comprised of three regions: 1) an Nterminal cell-membrane-anchor region; 2) a large extension region consisting of tens to hundreds of tandem Bacterial Immunoglobulin-like (BIg) domains; and 3) a C-terminal region containing domains responsible for adhesion and cohesion. Bioinformatic analysis of putative RTX adhesins in various bacterial species show that the first two regions are largely conserved, while the C-terminal regions contain a variety of currently unidentified ligand-binding domains, or domain homologues whose ligands are not yet known. Here we illustrate the variability in C-terminal regions having solved the structure of RTX adhesin ligand-binding domains from two divergent bacteria: the oil-eating bacterium Marinobacter hydrocarbonoclasticus and the opportunistic pathogen Aeromonas hydrophila. The structure of a sugarbinding domain from the $M$. hydrocarbonoclasticus adhesin was solved by molecular replacement as a PA14-like $\beta$-sandwich (1.0-Å resolution). The protein uses $\mathrm{Ca}^{2+}$ to coordinate a glucose molecule via the $\mathrm{C} 1$ and $\mathrm{C} 2$ hydroxyl groups of the sugar. The importance of similar domains in previously studied multispecies biofilms implicates this sugar-binding domain in cohesion between microorganisms, either through binding to surface glycans or exopolysaccharides. The adhesin from A. hydrophila was found to contain a von Willebrand Factor A-like (vWFA) domain within its C-terminal region. The structure was solved using SAD phasing, with $\mathrm{Ca}^{2+}$ as the heavy atom (1.4- $\AA$ resolution). A Rossman fold made up the majority of the structure, but an unexpected $\beta$-sandwich domain was found inserted between secondary structure elements. The predicted metal-ion-dependent adhesive site (MIDAS) contained a calcium ion that was bound to the C-terminus of another vWFA domain within the crystal. The similarity of this domain to known extracellular-matrix-binding proteins, like integrin, leads us to hypothesize that this protein may be involved in adhesion of the pathogen to host tissue. Using the structures of these recombinantly-expressed proteins in concert with a series of biochemical assays, we have begun to identify the ligands for each domain, and understand how differences in domain complement reflect the organism-specific substrate-binding needs.

Funded by NSERC 8tdrv@queensu.ca 